\title{
Journal of Stem Cell and Regenerative Biology
}

\section{Stem Cells in Translation versus Innovative Biomaterials and /or Nanomaterials}

\author{
Bipasha Bose*
}

Centre for Stem Cells and Regenerative Medicine, Yenepoya Research Centre, Yenepoya University, Karnataka, India

*Corresponding author: Bipasha, Bose. Centre for Stem Cells and Regenerative Medicine, Yenepoya Research Centre, Yenepoya University, Mangalore-575018, Karnataka, India; E-mail: Bipasha.bose@yenepoya.edu.in

Citation: Bose, B. Stem Cells in Translation versus Innovative Biomaterials and /or Nanomaterials. (2016) J Stem Cell Regen Biol 2(2): $94-96$

Received Date: July 11, 2016

Accepted Date: July 11, 2016

Published Date: July 15, 2016

DOI: $10.15436 / 2471-0598.16 .016$

\section{Stem Cells in Research}

Due to the "stemness" property of stem cells, stem cell research sounds very attractive. By "stemness" one means the plasticity to give rise to other cell types. Stemness, self-renewal and infinite proliferation potential of stem cells make them attractive targets for translational research in the modern times for generation of new tissues and organs for therapeutic purposes. Indeed, stem cell research involves looking into the genetic, epigenetic and other mechanisms related to stemness, infinite proliferation, and self-renewal. Biologists around the globe are busy either answering the fundamental properties of pluripotent and adult stem cells or cancer stem cells that could lead them to the ways of manipulating the stem cells for translation. In parallel, engineers/material scientists are also working globally to design various bio/nano materials that could be combined with the stem cell manipulation techniques for translation.

Of all the properties of stem cells that make them desirable candidates for translational research, stemness tops the list. Hence, various researchers worldwide are looking into the possibilities for enhancing the stemness of stem cells using various nanomaterials and biomaterials. Scientists all over the world have worked extensively on the multilineage differentiation potential of stem cells. Pluripotent stem cells have the ability to differentiate into all the lineages, while the adult stem cells have differentiation abilities into restrictive lineages. Hence, with regards to, the enhancement of differentiation potential various innovative biomaterials and nanomaterials can be explored, experimented and validated. The undesirable property of pluripotent stem cells is their teratoma formation potential. Hence, with regards to, the teratoma-forming potential, various nanomaterials can also be explored and validated that can eliminate the teratoma-forming cells.

In the case of regenerative stem cells, various methods of encapsulation, scaffolding ${ }^{[1]}$, or attachment of different nanomaterials is a right approach for enhancing various desirable properties, as well as, tissue engineering applications. Microengineering for obtaining differentiated human pluripotent stem cells on a chip is one of the approaches to enhance the functional properties of differentiated cells along with ensuring the microcirculation ${ }^{[2-8]}$. Contrarily, in the case of cancer stem cells (CSCs), nanomaterials might be helpful especially while targeting such cells or else for imaging. Also, various methods of mimicking the in vivo extracellular matrices using nanomaterials with a similar young modulus of elasticity provide stiffness and enhanced function to the regenerative stem cells.

In case of CSCs, one important phenomenon that is responsible for cancer metastasis and relapse has been revealed as epi- 
thelial to mesenchymal transition (EMT) of CSCs. Hence, innovative nano-biomaterial that can reverse this EMT into mesenchymal to epithelial transition (MET) could be a successful candidate in eliminating CSC population. Targeted killing of CSCs without toxicity to normal cells can be another strategy for designing novel nano-biomaterial.

\section{Stem Cells in Translation}

Advancement of the stem cell research in the translational direction towards organ and tissue replacement, drug testing and disease models are highly sought after. As far as the long-term goal of stem-cell research in translation is concerned, stem cells of which majority are bone marrow mesenchymal stem cells have already been into clinical trials/ and some of them already in clinics for addressing various disease conditions. Examples of such stem cell clinical trials registered in the NIH clinical trials registry (www.clinicaltrials.gov) that are currently recruiting patients are autologous bone marrow derived stem cells for the treatment of retinal and optic nerve disease/damage involving various diseased conditions of the eye such as retinal disease, macular degeneration, hereditary retinal dystrophy, optic nerve disease and glaucoma (Stem Cell Ophthalmology Treatment study-SCOT). Autologous Adipose-derived stem cell study for safety and efficacy in chronic pulmonary obstructive disease (COPD) patients is another example of such clinical trial under NIH clinical trial registry that is also recruiting patients.

For orthopedic conditions, it is important to have a combination of stem cells and a biomaterial. For example, a clinical trial registered with NIH registry for the reconstruction of the jaw bone that uses a biomaterial and autologous bone marrow derived stem cells is also currently recruiting patients. All the translational approaches using stem cell therapeutics need to address the generation of stem cell niche thereby mimicking the in vivo situations. Various components of stem cell niche involve heterologous cell-cell interaction for bi-directional signaling ${ }^{[9,10]}$, secreted and membrane-bound factors such as Wnt, SCF, Notch and chemokines directly binding to the surface receptors thereby regulating cell fate ${ }^{[11,12]}$, self-renewal and cell polarity, immunological parameters ${ }^{[13,14]}$ physical parameters ${ }^{[15-17]}$ and hypoxia ${ }^{[18-20]}$.

Are Stem Cells Alone or in Combination with Nanomaterials and/or Biomaterials a Better Approach for Cell Cell Therapy?

Stem cells alone have been used for the amelioration of various diseases in animal models such as Parkinson's disease ${ }^{[21]}$, diabetes ${ }^{[22]}$, spinal cord injury ${ }^{[23]}$ and much more. However, the downside of using stem cells alone for cell therapy purposes involves the chances of dilution of the engraftment of such cells and need for repeated stem cell transplantations. As stem cells inside the body occupy a cellular niche involving extracellular matrix (ECM), signaling molecules, immune environment, it is important to provide the stem cells with in vivo like situations for cell therapy applications along with a sustained engraftment. ECM not only provides the stiffness and support to the cell milieu but also maintains homeostasis by regulating the local $\mathrm{pH}$, oxygen tension, concentration of growth factor and receptors, thereby ensuring the survival of the stem cells against cellular stress and environmental factors. Hence, while designing a stem cell-based cell therapy product, it is always advantageous to develop the entire milieu using a combination of stem cells and ECM made of various biomaterials.

Innovative biomaterials need to be designed such that it these are biocompatible, have excellent mechanical properties, should be superporous and able to integrate into the biological environment and immunologically competent. Hence, biomaterials designed for specific cell therapy application will depend on the cell type and cellular niche. Various biocompatible biomaterials include silk, collagen and alginate hydrogels ${ }^{[24]}$. Classes of hydrogels that can be harnessed for cell therapy applications are the ones having excellent mechanical properties, stimuli-sensitive hydrogels and self-assembled hydrogels. Growth factor mediated assembly of hydrogels ${ }^{[25]}$ can also be useful for the localized delivery and efficient engraftment of stem cells. Stimuli-sensitive hydrogels can be used for triggering the activity of transplanted stem cells in the graft region depending on the local stimuli.

Composite nano-biomaterials can also be designed for enhancing the role of transplanted stem cells. Also, for CSCs, composite nano-biomaterials can be used as drug delivery vehicles targeting CSCs.

\section{Challenges in Regenerative Medicine versus Innovative Nanomaterials and/or Biomaterials}

As prime criteria in regenerative medicine involves controlled differentiation, immune reactions, sustained engraftment of stem cells and engineering complex structures, recent progress in the area of nanotechnology/biomaterials has led to the development of such multifunctional bio-nanomaterials. However, controlling the microenvironment for sustained stem cell fate upon transplantation of stem cell-loaded nano/biomaterial decides the therapeutic success of such novel nano/biomaterials in regenerative medicine. Most importantly, interactions between stem cells and nano/biomaterials are not completely understood. Hence, the most significant challenge is the critical evaluation of the effects of the shape, surface morphology and chemical functionality of nano/ biomaterial on stem cells.

Another challenge is the minuscule nature of the nano/biomaterial and possible induction of toxic properties of stem cells upon stem cell loading. Hence, ensuring the safety in regenerative medicine application using stem cells and nano/biomaterial needs to be well addressed before using such tools in the clinics. Safe injectible hydrogels are one such option in regenerative medicine ${ }^{[26]}$.

\section{Conclusions}

As designing of nano/biomaterial is mostly carried out by the material engineers, there needs to be a great understanding of the biology before using them for regenerative medical applications. The biggest advantage of designing novel nano/biomaterial is their flexibility in design, as most of them are prepared by chemical synthesis. Detailed understanding of nano/biomaterial stem cell interactions will ensure appropriate design of novel nano/biomaterial for regenerative medicine and other applications in biology 
and medicine. However, rigorous in vitro and in vivo validation of novel nano/biomaterial is mandatory before recommending such nano/biomaterial for clinical applications.

\section{References}

1. Wang, L., Xu, C., Zhu, Y., et al. Human induced pluripotent stem cell-derived beating cardiac tissues on paper. (2015) Lab Chip 15(22): 42834290 .

2. Marsano, A., Conficconi, C., Lemme, M., et al. Beating heart on a chip: a novel microfluidic platform to generate functional 3D cardiac microtissues. (2016) Lab Chip 16(3): 599-610.

3. Giobbe, G.G., Michielin, F., Luni, C., et al. Functional differentiation of human pluripotent stem cells on a chip. (2015) Nat Methods 12(7): 637-640.

4. Grosberg, A., Alford, P.W., McCain, M.L., et al. Ensembles of engineered cardiac tissues for physiological and pharmacological study: Heart on a chip. (2011) Lab Chip 11(24): 4165-4173.

5. Huh, D., Matthews, B.D., Mammoto, A., et al. Reconstituting organ-level lung functions on a chip. (2010) Science 328(5986): $1662-1668$.

6. Domansky, K., Inman, W., Serdy, J., et al. Perfused multiwell plate for 3d liver tissue engineering. (2010) Lab Chip 10(1): 51-58.

7. Tsai, M., Kita, A., Leach, J., et al. In vitro modeling of the microvascular occlusion and thrombosis that occurin hematologic diseases using microfluidic technology. (2012) J Clin Invest 122(1): 408-418.

8. Chau, L.T., Rolfe, B.E., Cooper-White, J.J. A microdevice for the creation of patent, three-dimensional endothelial cell-based microcirculatory networks. (2011) Biomicrofluidics 5(3): 34115-3411514.

9. Lo, Celso, C., Fleming, H.E., Wu, J.W., et al. Live-animal tracking of individual haematopoietic stem/progenitor cells in their niche. (2009) Nature 457(7225): 92-96.

10. Kiel, M.J., Yilmaz, O.H., Iwashita, T., et al. SLAM family receptors distinguish hematopoietic stem and progenitor cells and reveal endothelial niches for stem cells. (2005) Cell 121(7): 1109-1121.

11. Katayama, Y., Battista, M., Kao, W.M., et al. Signals from the sympathetic nervous system regulate hematopoietic stem cell egress from bone marrow. (2006) Cell 124(2): 407-421.

12. van Es, J.H. Sato, T., van de Wetering, M., et al. Dll1+ secretory progenitor cells revert to stem cells upon crypt damage. (2012) Nat Cell Biol 14(10): 1099-1104.

13. Jaiswal, S., Jamieson, C.H., Pang, W.W., et al. CD47 is upregulated on circulating hematopoietic stem cells and leukemia cells to avoid phagocytosis. (2009) Cell 138(2): 271-285.

14. Fujisaki, J., Wu, J., Carlson, A.L., et al. In vivo imaging of Treg cells providing immune privilege to the haematopoietic stem-cell niche. (2011) Nature 474(7350): 216-219.

15. McBeath, R., Pirone, D.M., Nelson, C.M., et al. Cell shape, cytoskeletal tension, and RhoA regulate stem cell lineage commitment. (2004) Dev Cell 6(4): 483-495.

16. Holst, J., Watson, S., Lord, M.S., et al. Substrate elasticity provides mechanical signals for the expansion of hemopoietic stem and progenitor cells. (2010) Nat Biotechnol 28(10): 1123-1128.

17. Gilbert, P.M., Havenstrite, K.L., Magnusson, K.E., et al. Substrate elasticity regulates skeletal muscle stem cell self-renewal in culture. (2010) Science 329(5995): 1078-1081.

18. Kimura, W., Sadek, H.A. The cardiac hypoxic niche: emerging role of hypoxic microenvironment in cardiac progenitors. (2012) Cardiovasc Diagn Ther 29(4): 278-289.

19. Muscari, C., Giordano, E., Bonafè, Fet., al. Priming adult stem cells by hypoxic pretreatments for applications in regenerative medicine. (2013) J Biomed Sci 20: 63.

20. Forristal, C.E., Winkler, I.G., Nowlan, B., et al. Pharmacologic stabilization of HIF-1alpha increases hematopoietic stem cell quiescence in vivo and accelerates blood recovery after severe irradiation. (2013) Blood 121(5): 759-769.

21. Zhang, J., Wang, X., Li, J., et al. The Preclinical Research Progress of Stem Cells Therapy in Parkinson's Disease. (2016) Biomed Res Int 2016: 5683097.

22. Pipeleers, D., Robert, T., De, Mesmaeker., et al. Concise Review: Markers for Assessing Human Stem Cell-Derived Implants as $\beta$-Cell Replacement in Type1 Diabetes. (2016) Stem Cells Transl Med : 0187.

23. Zhou HL, Zhang XJ, Zhang MY. Transplantation of Human Amniotic Mesenchymal Stem Cells Promotes Functional Recovery in a Rat Model of Traumatic Spinal Cord Injury. (2016) Neurochem Res.

24. Kopecek, J. Hydrogel biomaterials-a smart future? (2007) Biomaterials 28(34): 5185-5192.

25. Yamaguchi, N., Zhang, L., Chae, B.S., et al. Growth factor mediated assembly of cell receptor-responsible hydrogels. (2007) J Am Chem Soc 129(11): 3040-3041.

26. Sepantafar, M., Maheonnagsh, R., Mohammadi, H., et al. Stem cells and injectible hydrogels: Synergistic therapeutics in myocardial repair. (2016) Biotechnol Adv 34(4): 362-367.

Ommega Online Publisher

Journal of Stem Cell \& Regenerative Biology

Short Title : J Stem Cell Regen Biol
ISSN: 2471-0598

E-mail : stemcells@omegaonline.org

website: www.ommegaonline.org 